\title{
Barriers to Recruiting and Engaging End-Users in Large-Scale Digital Health \& Wellbeing Technologies and Services
}

\author{
Siobhan O'Connor, Frances S. Mair, \\ Kate O'Donnell \\ General Practice \& Primary Care, \\ Institute of health \& Well-Being \\ University of Glasgow \\ s.oconnor.1@research.glac.uk \\ FirstName.LastName@glasgow.ac.uk
}

\author{
Marilyn McGee-Lennon, \\ Matt-Mouley Bouamrane \\ Dept. of Computer \& \\ Information Science, \\ University of Strathclyde, \\ Scotland, \\ FirstName.LastName@strath.ac.uk
}

\begin{abstract}
Health and social care services in the U.K. are increasingly looking towards digital technologies and services as a way to support those with chronic illness to better manage their disease and to allow older people to continue living independently for longer. The Delivering Assisted Living Lifestyles at Scale (dallas) programme aims to address these two issues. However implementing a large-scale digital health innovation programme is a hugely complex process. This paper reports preliminary qualitative findings from the analysis of project documentation and interviews with the four consortia of the dallas programme. It presents an overview of the difficulties experienced when engaging and attempting to recruit individuals to participate in the dallas related services. A number of barriers - such as the use of co-design methodologies, branding and partnership constraints and unsustainable recruitment targets - hindered initial engagement strategies when trying to reach and enrol large numbers of end-users. The recruitment strategies of the consortia within this digital health innovation programme have hence continued to evolve in order to adapt to the emerging realities on the ground.
\end{abstract}

eHealth, Integrated Delivery of Health Care, National Health Programs, Qualitative Research.

\section{INTRODUCTION}

National health and social care services are facing many important challenges due to our aging population [1]. Digital health technologies and services are currently being developed with the aim of reducing the burden placed on the National Health Service (NHS) by the wide prevalence of chronic illnesses such as heart disease, diabetes, cancer, and asthma among others [2].

Currently, digital health technologies are often evaluated using either pilot studies or randomised controlled trials to judge whether they are effective in improving clinical outcomes or reducing health service utilization. Even though eHealth initiatives can produce benefits in certain settings, scaling up new technologies and services within complex healthcare systems can face significant barriers. The factors at play within such large scale deployment of technologies are still very much insufficiently understood [3].
Therefore, studying and identifying factors which promote or hinder digital health solutions implementations at scale is therefore essential if one is to hope making progress in the field in future.

Delivering Assisted Living Lifestyles at Scale (dallas) is a three-year digital innovation programme funded by the UK Technology Strategy Board which aims to demonstrate how assisted living and other technologies can be used to promote health and wellbeing and improve people's lifestyles [4].

In tandem, the programme seeks to establish a consumer market for digital health and social care products and services by promoting the design, development and roll-out of these technologies at scale.

Dallas comprises four service-led consortia, called 'Living It Up', 'More Independent', 'Year Zero' and 'iFocus', which consist of a range of organisations including NHS Trusts, local authorities, voluntary organisations, industry and academia. 
These four communities are designing and rolling out a range of digital health and social care products and services such as personal electronic health records, telehealth and telecare solutions, assisted living technologies and mobile health applications among others.

The purpose of our research is to both assess the impact of the dallas programme as well as examining the factors which affect recruitment in a large-scale digital health programme.

\section{METHODS \& PRELIMINARY FINDINGS}

\subsection{Methods Overview}

We are using a qualitative framework and thematic approach [5] to examine the barriers experienced by each dallas community when recruiting participants. Data collection and analysis involves a combination of documentation analysis (including consortia project plans and quarterly progress reports) along with qualitative interviews of key members from the four dallas consortias, involved in the development of the consortia implementation strategies and services.

\subsection{Preliminary Findings}

Preliminary data analysis suggest a number of sociotechnical barriers [6,7] throughout the process of engaging and recruiting participants.

- A key factor which hindered recruitment initially was the use of a co-design methodology to create a personalised digital portal in collaboration with end-users. Users were thus exposed to a series of prototypes that emerged from this collaborative design process as many of the features and functions of the online portal developed. This meant many active participants became frustrated when trying to use the unfinished platform and potential users were unwilling to engage until the digital portal went live. These issues prevented the scale-up of recruitment efforts to reach larger audiences which are only now getting underway on completion of the digital portal.

- Unsustainable recruitment strategies were also utilised during the initial phases of the dallas programme. For example face-to-face strategies were adopted to engage directly with individuals in the community. However this approach proved too slow and consumed valuable time and manpower resources. Therefore community engagement events to recruit individuals, while useful, are unsuitable to support large scale recruitment efforts.

- Equally, some branding exercises slowed recruitment efforts as many of the new digital products and services were not marketed as being affiliated with well-known healthcare organisations or recognised professional bodies. Potential users were not familiar with dallas and hence initial consumer confidence and engagement with this new brand was low.

- Partnerships were also established with organisations that had access to large membership databases but some of these were not directly aligned with the dallas vision and so accessing their members directly became problematic.

These barriers have meant that recruitment to the dallas programme remains challenging to date. --

\section{DISCUSSION}

Deploying any new digital product or service in healthcare is usually a long and complex process and one that requires careful design and planning in order to ensure a successful implementation [8]. The first step in this process is often to start with engaging with a range of stakeholders such as patients, carers, healthcare providers and others with a view to collecting system and service requirements as well as achieving a level of engagement and 'buy-in' [9]. However, substantial difficulties can often arise when attempting to recruit individuals to new digital health services [10]. Unsurprisingly the diverse and intricate nature of a large-scale digital innovation programme such as dallas has meant that it too has experienced a number of barriers to recruitment, which stem in part from how the consortia have developed and implemented some of their digital products and services.

The UK Medical Research Council complex intervention framework recommends that new health interventions are evaluated in depth before they are implemented and scaled up [11]. The MRC guidance also suggests the use of theoretical frameworks to aid our understanding of the range of factors which affect implementation processes in practice. We intend to further refine our data analysis in future work and use Normalization Process Theory (NPT) [12], as it has been used to explore a range of complex and large scale eHealth implementations $[3,9,13,14]$. NPT could hence provide a useful lens through which to understand how recruitment to large scale digital health initiatives unfolds. The dallas programme is still progressing and as such recruitment strategies are continuously evolving to meet the demands of this large-scale digital health innovation programme. Key barriers in the recruitment process are and will continue to emerge. Although the dallas communities are addressing recruitment issues and have reported positive engagement, it is still too early to assess whether the diverse engagement approaches will ultimately be sufficient to achieve the programme initial recruitment targets. 


\section{REFERENCES}

[1] Barnett, K.; Mercer, S. W.; Norbury, M.; Watt, G.; Wyke, S. \& Guthrie, B. Epidemiology of multimorbidity and implications for health care, research, and medical education: a crosssectional study. The Lancet, 2012, 380, 37-43

[2] Bouamrane, M.-M.; Osbourne, J. \& Mair, F. Understanding the implementation and integration of remote tele-health services; an overview of Normalization Process Theory. Pervasive Computing Technologies for Healthcare (PervasiveHealth), 2011 5th International Conference on, 2011, 300-307, IEEE

[3] Mair, F.S., May, C., O'Donnell, C., Finch, T., Sulilvan, F. and Murray, E. 2012. Factors that promote or inhibit the implementation of e-health systems: an explanatory systematic review. Bulletin of the World Health Organisation, 90(5), 357-364.

[4] McGee-Lennon M, Bouamrane M-M, Barry S et al. Evaluating the Delivery of Assisted Living Lifestyles at Scale (dallas) Proceedings of Design \& Implementation of Independent \& Assisted Living Technology, 26th BCS Conference on Human Computer Interaction, HCl2012 - People \& Computers XXVI, UK, 2012.

[5] Ritchie, J., \& Spencer, L. (2002). Qualitative data analysis for applied policy research. The Qualitative Researcher's Companion, 305-329.

[6] Harrison MI, Koppel R, Bar-Lev S: Unintended consequences of information technologies in health care - an interactive sociotechnical analysis. J Am Med Inform Assoc 2007, 14(5):542-549.

[7] Novak LL, Holden RJ, Anders SH, Hong JY, Karsh BT: Using a sociotechnical framework to understand adaptations in health IT implementation. Int J Med Inform 2013, 82(12):e331—e344
[8] van Gemert-Pijnen, J. E., Nijland, N., van Limburg, M., Ossebaard, H. C., Kelders, S. M., Eysenbach, G., \& Seydel, E. R. (2011). A holistic framework to improve the uptake and impact of eHealth technologies. Journal of Medical Internet Research, 13(4).

[9] Bouamrane, MM and Mair, F. Implementation of an integrated preoperative care pathway and regional electronic clinical portal for preoperative assessment. BMC Medical Informatics and Decision Making 14 (1), 93

[10] Sanders, C., Rogers, A., Bowen, R. et al. (2012). Exploring barriers to participation and adoption of telehealth and telecare within the Whole System Demonstrator trial: a qualitative study. BMC Health Serv. Res. 2012 Jul 26; $12: 220$

[11] Craig, P., Dieppe, P., Macintyre, S., Michie, S., Nazareth, I., \& Petticrew, M. (2008). Developing and evaluating complex interventions: the new Medical Research Council guidance. British Medical Journal, 337.

[12] May C, Finch T. Implementation, embedding, and integration: an outline of normalization process theory. Sociology 2009;43:535-54

[13] Bouamrane, M.-M. \& Mair, F. A study of general practitioners' perspectives on electronic medical records systems in NHSScotland. BMC Medical Informatics and Decision Making, 2013, 13:58.

[14] Bouamrane, M.-M. \& Mair, F. A qualitative evaluation of general practitioners' views on protocol-driven eReferral in Scotland. BMC Medical Informatics and Decision Making. 2014; 14: 30 